\title{
Weather Variability, Socioeconomic Factors, and Pneumonia in Children Under Five-Years Old — Bangladesh, 2012-2016
}

\author{
Mohammad Zahid Hossain ${ }^{1,2}$; Shilu Tong ${ }^{1,3,4,5}$ Hilary Bambrick ${ }^{1}$; Md Alfazal Khan ${ }^{6}$; Wenbiao Hu ${ }^{1, \#}$
}

\section{Summary}

What is already known on this topic?

Different socioecological factors were associated with childhood pneumonia in Bangladesh. However, previous studies did not assess spatial patterns, and socioecological factors and spatial variation have the potential to improve the accuracy and predictive ability of existing models.

\section{What is added by this report?}

The spatial random effects were present at the district level and were heterogeneous. Average temperature, temperature variation, and population density may influence the spatial pattern of childhood pneumonia in Bangladesh.

What are the implications for public health practice?

The study results will help policymakers and health managers to identify the vulnerable districts, plan further investigations, help to improve proper resource allocation, and improve health interventions.

Pneumonia is one of the leading causes of mortality and morbidity in children aged under five years in Bangladesh. This study aimed to identify the association between weather, social factors and childhood pneumonia and identify the spatial variation of the disease. A Bayesian spatial Poisson regression model with a conditional autoregressive prior structure was developed to quantify the association between childhood pneumonia and socioecological factors and identify the spatial variation. The study results suggested that a $1{ }^{\circ} \mathrm{C}$ increase in monthly temperature and monthly temperature variation may increase the monthly associated log relative risk (RR) of childhood pneumonia by 1.161 [95\% credible interval $(\mathrm{CrI})$ : 1.013-1.429] and 1.463 (95\% CrI: 1.170-1.839), respectively. However, the population density was inversely related with pneumonia risk (RR: 0.996, CrI: 0.994-0.998). Socioecological factors may influence the spatial pattern of childhood pneumonia, and the spatial random effects were heterogeneous.
The study was conducted in Bangladesh, which is located in the northeastern part of South Asia. Bangladesh is divided into 8 administrative divisions and 64 districts. Monthly data on under-5-years pneumonia were extracted from the District Health Information System Version 2 of the Directorate General of Health Services (DGHS) under the Ministry of Health and Family Welfare of Bangladesh from January 2012 to December 2016 (1). The pneumonia cases were diagnosed according to the World Health Organization pneumonia guidelines (2). The under-five-years population data at the district level were collected from the latest national population and household census (3). The sociodemographic data (percentage of education and internet use) at the district level were collected from socioeconomic and demographic reports (national series, volume-4) from the same census. The poverty data for each district was obtained from the Household Income and Expenditure Survey 2016 (4).

Climate data (temperature and rainfall) were obtained from the National Environmental Satellite, Data and Information service (https://www7.ncdc. noaa.gov/CDO/cdoselect.cmd?datasetabbv=GSOD), which is publicly available and widely used in previous studies (5-6). Poisson regression models in a Bayesian framework were developed for pneumonia cases at the district level. These models assume that the observed counts of childhood pneumonia cases $\left(\mathrm{O}_{k}\right)$ for the $k$ th district $(k=1 \cdots 64)$ follow a Poisson distribution with mean $\mu_{k}$ :

$$
\begin{gathered}
\mathrm{O}_{k} \sim \text { Poisson }\left(\mu_{k}\right) \\
\log \left(\mu_{k}\right)=\log \left(\mathrm{E}_{k}\right)+\theta_{k}
\end{gathered}
$$

where $\mathrm{E}_{k}$ (the expected number of cases in District ${ }_{k}$ ) is an offset to control population size and $\theta_{k}$ is the associated $\log$ RR.

Prior to this analysis, we examined multicollinearity among the different covariates but did not find sufficiently strong associations to warrant exclusion or other treatment of any variables (Supplementary Table S1, available in http://weekly.chinacdc.cn). As a consequence, a total of 6 models were developed 
(Supplementary Material, available in http://weekly. chinacdc.cn). The model which incorporated all socioecological covariates with both structured and unstructured random effects were selected for the final analysis.

The expected $\log$ relative risk $\theta_{k}$ was represented as follows:

$$
\begin{aligned}
\theta_{k}= & \alpha+\left(\operatorname{Temp}_{k}\right) \beta_{1}+\left(\operatorname{Tempva}_{k}\right) \beta_{2}+\left(\operatorname{Rain}_{k}\right) \beta_{3} \\
& +\left(\operatorname{Edu}_{k}\right) \beta_{4}+\left(\operatorname{Int}_{k}\right) \beta_{5}+\left(\operatorname{povi}_{k}\right) \beta_{6} \\
& +\left(\operatorname{pop}_{k}\right) \beta_{7}+\mathrm{u}_{k}+\mathrm{v}_{k}
\end{aligned}
$$

where $\alpha$ is a constant; $\beta_{1}$ is the coefficient for temperature, $\beta_{2}$ is the coefficient for temperature variation, $\beta_{3}$ is the coefficient for rainfall, $\beta_{4}$ is the coefficient for percentage of education at the district level, $\beta_{5}$ is the coefficient for percentage of internet user at the district level, $\beta_{6}$ is percentage of poverty at the district level, and $\beta_{7}$ is the population density per square kilometer; $\mathrm{v}_{k}$ is a spatially unstructured random effect that is assumed to be normally distributed with mean zero and variance $\sigma_{v}{ }^{2}$ and $\mathrm{u}_{k}$ is the spatially structured random effect that was modeled using a conditional autoregressive (CAR) prior $\mathrm{u}_{k} \sim \mathrm{N}\left(\overline{\mathrm{u}}_{\sim k}, \sigma_{u}{ }^{2} / \mathrm{n}_{k}\right)$, where $\sim \mathrm{k}$ denotes the neighbors of the $k$ th district based on a simple adjacency matrix and $\mathrm{n}_{k}$ is the corresponding number of neighbors (7). WinBUGS software (version 1.4.3, MRC Biostatistics Unit, Cambridge, and Imperial College School of Medicine, London) was used to fit the Bayesian Poisson regression models. In the Markov chain Monte Carlo analysis, a 30,000 iteration "burn-in" was followed by 100,000 iteration sample collection. In every case, the Monte Carlo error was $<5 \%$ of the overall standard deviation, indicating sufficient iterations of the model had been run after convergence. Model comparison was performed using the Deviance Information Criterion (DIC). Best fitted models were indicated by smaller DIC values (8).

The mean monthly number of pneumonia cases in children $<5$ years was 747.82 . The mean monthly temperature, temperature variation, and rainfall were $30.97{ }^{\circ} \mathrm{C}, 3.63{ }^{\circ} \mathrm{C}$, and $164.54 \mathrm{~mm}$, respectively. Among the social factors, the mean percentages of education, internet use, poverty (per 100 population) and $<5$ years children density (per square kilometer) were $54.66 \%, 0.62 \%, 24.45 \%$, and 117.72 , respectively, at the district level (Supplementary Table S2, available in http://weekly.chinacdc.cn). The average monthly temperature was higher in the western region, while the monthly temperature variation was higher in most of the hilly areas located in the southern part of the country and two districts (Bhola and
Pirojpur) of the coastal region of Bangladesh. The distributions of higher monthly average rainfall were scattered in different regions. Inclusion of spatial autocorrelation in the model was important. The model which included both structured and unstructured random effects had the smallest DIC (665.47 and 13,773.00 for models with and without random effects, respectively) (Supplementary Table S3, available in http://weekly.chinacdc.cn). The highest RRs were observed in the southeastern part (Rangamati district) and southern part (Pirojpur district) of the country (Figure 1). Supplementary Table S4 (available in http://weekly.chinacdc.cn) shows the list of districts with the higher RR.

Figure 2 depicts the distribution of spatial random effects (structured heterogeneity) of pneumonia in Bangladesh. The districts with darker color (red color) had relatively high spatial variation. These districts with high spatial variation might have some unknown factors that may have had effects on the incidence of childhood pneumonia but that we did not consider in the models (e.g., incomplete measurement of variables, lack of geocoding, and generalization of geographic features).

Our study results suggested that a rise of $1{ }^{\circ} \mathrm{C}$ average monthly temperature and temperature

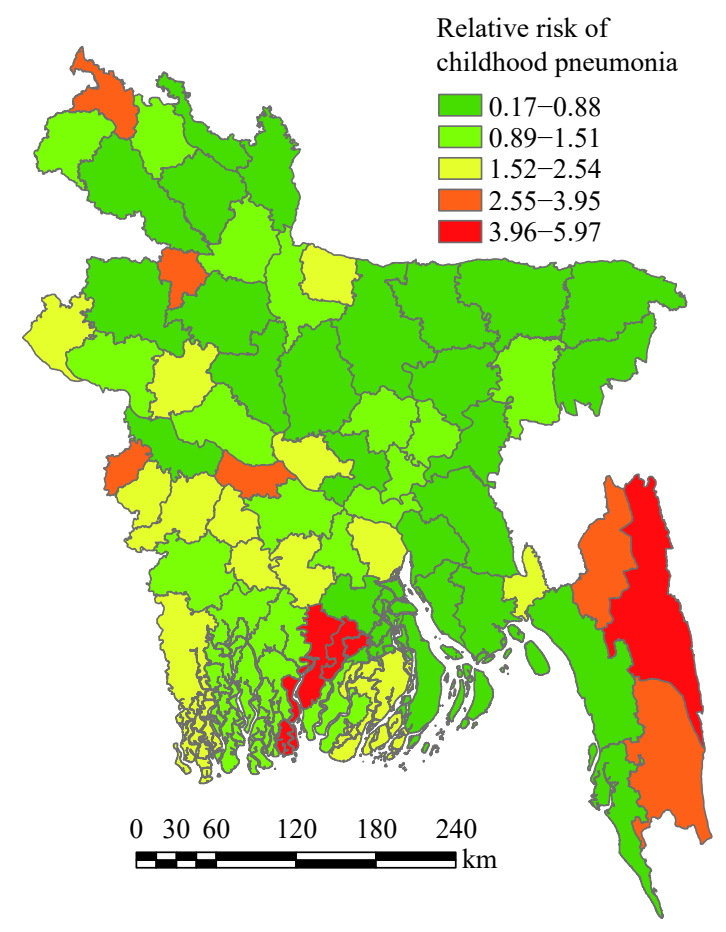

FIGURE 1. Posterior estimated Relative Risk of childhood pneumonia at the district level of Bangladesh from 2012 to 2016. 


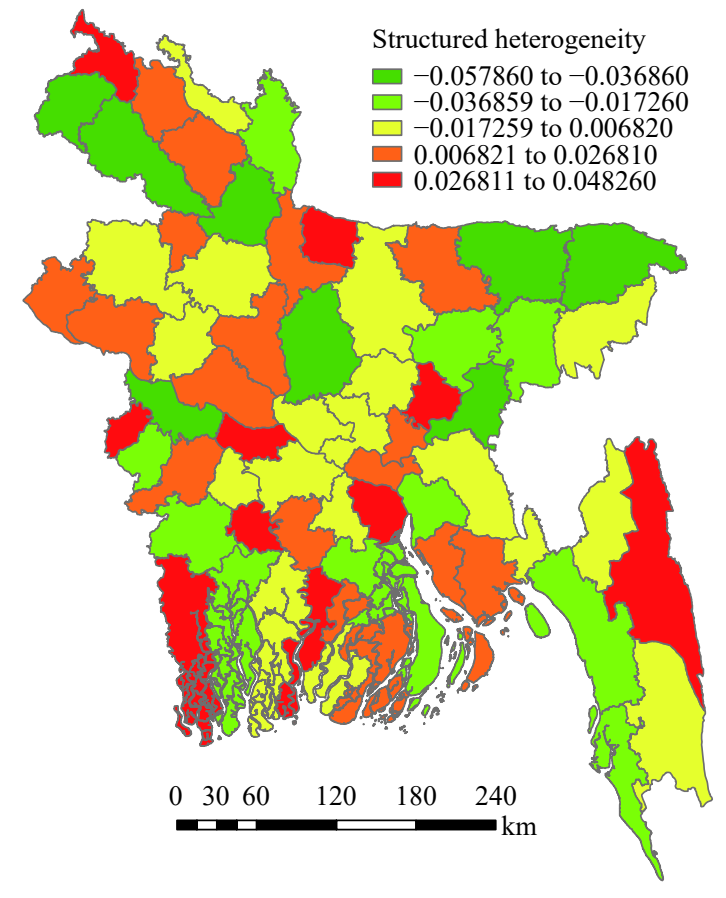

FIGURE 2. Spatial random effects of childhood pneumonia — Bangladesh, 2012-2016.

variation was associated with $\mathrm{RR}$ estimates of childhood pneumonia of $1.161 \quad(95 \%$ CrI: $1.012-1.428$ ) and 1.463 (95\% CrI: 1.169-1.838), respectively. The density of children under five years in population was negatively associated with pneumonia (RR: 0.996, 95\% CrI: 0.994-0.998) (Table 1).
Additionally, no significant associations were found between childhood pneumonia and rainfall, education, internet use, or poverty since the corresponding $95 \%$ CrIs for the RR of each factor included 1 .

\section{DISCUSSION}

In young children, the thermoregulation system is not yet matured and makes the children more vulnerable to temperature variation. This study describes the spatial pattern of childhood pneumonia and their socioecological factors in Bangladesh. Identifying the spatial variation of childhood pneumonia and important socioecological determinants can help target high-risk communities with evidence-based effective preventative measures.

Mapping of the spatially structured random effects indicated the spatial variation after controlling socioecological factors and spatial autocorrelation in the model. The Bayesian CAR model included unknown parameters as random effects, which incorporated the spatially correlated random effects (9). This approach can account for the residual variability resulting from spatial variation in effects that were not included in the models. The districts containing higher spatial random effects or variation may have some other risk factors remaining after adjustment of socioecological factors and spatial correlation.

TABLE 1. Crude and adjusted RR of different socioecological factors in Children Under Five-Years Old — Bangladesh, 2012-2016.

\begin{tabular}{ccc}
\hline Variables & Crude RR (95\% Crl) & Adjusted RR (95\% Crl) \\
\hline Temperature $^{*}$ & $1.146(0.929-1.432)$ & $1.161(1.013-1.429)$ \\
Temperature $^{\dagger}$ & $1.730(1.694-1.763)$ & $1.529(1.503-1.555)$ \\
Temperature variability $^{*}$ & $1.821(1.376-2.491)$ & $1.463(1.170-1.839)$ \\
Temperature variability $^{\dagger}$ & $1.623(1.596-1.649)$ & $1.421(1.395-1.447)$ \\
Rainfall $^{*}$ & $1.000(0.999-1.002)$ & $1.000(0.999-1.002)$ \\
Rainfall $^{\dagger}$ & $1.0007(1.0006-1.0007)$ & $1.0001(1.0000-1.0002)$ \\
Population density $^{*}$ & $0.995(0.994-0.997)$ & $0.996(0.994-0.998)$ \\
Population density $^{\dagger}$ & $0.9943(0.9942-0.9945)$ & $0.9959(0.9958-0.9961)$ \\
Education $^{*}$ & $0.979(0.956-1.004)$ & $0.986(0.969-1.005)$ \\
Education $^{\dagger}$ & $0.978(0.977-0.979)$ & $0.9853(0.984-0.986)$ \\
Poverty $^{*}$ & $1.008(0.996-1.020)$ & $1.000(0.988-1.010)$ \\
Poverty $^{\dagger}$ & $1.009(1.009-1.010)$ & $1.002(1.002-1.003)$ \\
Internet $^{*}$ & $0.844(0.661-1.070)$ & $0.916(0.743-1.126)$ \\
Internet $^{\dagger}$ & $0.874(0.862-0.885)$ & $0.929(0.916-0.942)$
\end{tabular}

Abbreviations: RR=relative risk; Crl=credible interval.

* with heterogeneity ( $u$ and $v)$.

${ }^{\dagger}$ without heterogeneity ( $u$ and $\left.v\right)$. 
This study was subject to some limitations. First, in this study, we used data from monthly reports of the DGHS. This represented the number of patients that attended different levels of health facilities in Bangladesh for pneumonia treatment. However, there might be some patients in the community who did not attend any health facilities and who received treatment from village doctors or spiritual healers, especially in the rural areas. Therefore, there was a chance of measurement and information biases. Second, the unit of analysis was at the group level rather than at the individual level, so the results may be prone to the ecological fallacy.

The findings of this study could help policymakers better understand that childhood pneumonia has a heterogeneous spatial pattern and that socioecological factors may play a significant role in describing this pattern.

Acknowledgements: The Management Information System, The Director General of Health Services, Ministry of Health, and Family Welfare, Bangladesh.

Funding: The Queensland University of Technology Postgraduate Research Award and Queensland University of Technology Higher Degree Research International Tuition Fee Sponsorship.

doi: $10.46234 / \mathrm{ccdcw} 2021.161$

\# Corresponding author: Wenbiao Hu,w2.hu@qut.edu.au.

\footnotetext{
School of Public Health and Social Work, Institute of Health and Biomedical Innovation, Queensland University of Technology, Brisbane, Queensland, Australia; ${ }^{2}$ Infectious Diseases Division, International Centre for Diarrhoeal Disease Research, Bangladesh, Mohakhali, Dhaka, Bangladesh; ${ }^{3}$ Shanghai Children's Medical Centre, School of Medicine, Shanghai Jiao Tong University, Shanghai, China; ${ }^{4}$ School of Public Health, Institute of Environment and Population Health, Anhui Medical University, Hefei, Anhui, China; ${ }^{5}$ Center for
}

Global Health, School of Public Health, Nanjing Medical University, Nanjing, Jiangsu, China; ${ }^{6}$ Health System and Population Studies Division, International Centre for Diarrhoeal Disease Research, Bangladesh, Mohakhali, Dhaka, Bangladesh.

Submitted: May 12, 2021; Accepted: July 13, 2021

\section{REFERENCES}

1. DGHSIMCInewsletter2017http://www.dghs.gov.bd/images/docs/IMCI/ IMCI_12_2017.pdf. [2020-11-13].

2. World Health Organization. Revised WHO classification and treatment of childhood pneumonia at health facilities-evidence summaries. 2014 https://apps.who.int/iris/rest/bitstreams/611120/retrieve. [2021-1-22].

3. Bangladesh Bureau of Statistics. Population and household census. 2011. http://www.bbs.gov.bd/site/page/47856ad0-7e1c-4aab-bd78-892733 bc06eb/Population-and-Housing-Census. [2021-2-18].

4. Bangladesh Bureau of Statistics, Statistics and Informatics Division, Ministry of Planning, Government of the People's Republic of Bangladesh. Bangladesh-household income and expenditure survey. 2016. expenditure survey. 2016. https://www.academia.edu/37874731/ Preliminary_Report_on_Household_Income_and_Expenditure_Survey_ 2016_BANGLADESH_BUREAU_OF_STATISTICS_BBS_STATISTI CS_AND_INFORMATICS_DIVISION_SID. [2020-9-17].

5. Gullón P, Varela C, Martínez EV, Gómez-Barroso D. Association between meteorological factors and hepatitis A in Spain 2010-2014. Environ Int 2017;102:230 - 5. http://dx.doi.org/10.1016/j.envint.2017. 03.008.

6. Thomson MC, Connor SJ, D'Alessandro U, Rowlingson B, Diggle P, Cresswell $M$, et al. Predicting malaria infection in Gambian children from satellite data and bed net use surveys: the importance of spatial correlation in the interpretation of results. Am J Trop Med Hyg 1999;61(1):2 - 8. http://dx.doi.org/10.4269/ajtmh.1999.61.2.

7. Hu WB, Clements A, Williams G, Tong SL, Mengersen K. Spatial patterns and socioecological drivers of dengue fever transmission in Queensland, Australia. Environ Health Perspect 2012;120(2):260 - 6. http://dx.doi.org/10.1289/ehp.1003270.

8. Duncan EW, White NM, Mengersen K. Spatial smoothing in Bayesian models: a comparison of weights matrix specifications and their impact on inference. Int J Health Geogr 2017;16:47. http://dx.doi.org/10. 1186/s12942-017-0120-x

9. Best N, Richardson S, Thomson A. A comparison of bayesian spatial models for disease mapping. Stat Methods Med Res 2005;14(1):35 - 59. http://dx.doi.org/10.1191/0962280205sm388oa. 


\section{Supplementary Material}

\section{Statistical models}

As a consequence, a total of 6 models were developed. Model I included only ecological measures (temperature, temperature variation, and rainfall) as explanatory variables; Model II included only social factors (education, internet use, population density, and poverty) as covariates; Model III included both ecological and social factors as explanatory variables; Model IV incorporated spatially structured random effects with all socioecological covariates; Model V incorporated spatially unstructured random effects with all socioecological covariates; Model VI incorporated both structured and unstructured random effects with all socioecological covariates.

The expected $\log$ relative risk $\theta_{k}$, for these models thus represented as follows:

$\theta_{k}=\alpha+\left(\right.$ Temp $\left._{k}\right) \beta_{1}+\left(\right.$ Tempva $\left._{k}\right) \beta_{2}+\left(\operatorname{Rain}_{k}\right) \beta$ Model I

$\theta_{k}=\alpha+\left(\mathrm{Edu}_{k}\right) \beta_{1}+\left(\operatorname{Int}_{k}\right) \beta_{2}+\left(\right.$ povi $\left._{k}\right) \beta_{3}+\left(\operatorname{pop}_{k}\right) \beta_{1}$ Model II

$\theta_{k}=\alpha+\left(\operatorname{Temp}_{k}\right) \beta_{1}+\left(\right.$ Tempva $\left._{k}\right) \beta_{2}+\left(\operatorname{Rain}_{k}\right) \beta_{3}+\left(\operatorname{Edu}_{k}\right) \beta_{4}+\left(\operatorname{Int}_{k}\right) \beta_{5}+\left(\right.$ povi $\left._{k}\right) \beta_{6}+\left(\right.$ pop $\left._{k}\right) \beta$ Model III

$\theta_{k}=\alpha+\left(\right.$ Temp $\left._{k}\right) \beta_{1}+\left(\right.$ Tempva $\left._{k}\right) \beta_{2}+\left(\operatorname{Rain}_{k}\right) \beta_{3}+\left(\mathrm{Edu}_{k}\right) \beta_{4}+\left(\operatorname{Int}_{k}\right) \beta_{5}+\left(\right.$ povi $\left._{k}\right) \beta_{6}+\left(\right.$ pop $\left._{k}\right) \beta_{7}+u$ Model IV

$\theta_{k}=\alpha+\left(\mathrm{Temp}_{k}\right) \beta_{1}+\left(\mathrm{Tempva}_{k}\right) \beta_{2}+\left(\operatorname{Rain}_{k}\right) \beta_{3}+\left(\mathrm{Edu}_{k}\right) \beta_{4}+\left(\mathrm{Int}_{k}\right) \beta_{5}+\left(\right.$ povi $\left._{k}\right) \beta_{6}+\left(\mathrm{pop}_{k}\right) \beta_{7}+\mathrm{v}$ .Model V $\theta_{k}=\alpha+\left(\mathrm{Temp}_{k}\right) \beta_{1}+\left(\mathrm{Tempva}_{k}\right) \beta_{2}+\left(\operatorname{Rain}_{k}\right) \beta_{3}+\left(\mathrm{Edu}_{k}\right) \beta_{4}+\left(\operatorname{Int}_{k}\right) \beta_{5}+\left(\right.$ povi $\left._{k}\right) \beta_{6}+\left(\right.$ pop $\left._{k}\right) \beta_{7}+\mathrm{u}_{k}+\mathrm{v}_{k} \ldots \ldots \ldots \ldots \ldots \ldots . . .$. Model VI where $\alpha$ is a constant, $\beta_{1}$ is the coefficient for temperature, $\beta_{2}$ is the coefficient for temperature variation, $\beta_{3}$ is the coefficient for rainfall, $\beta_{4}$ is the coefficient for percentage of education at district level, $\beta_{5}$ is the coefficient for percentage of internet user at district level, $\beta_{6}$ is percentage of poverty at district level, and $\beta_{7}$ is the population density per square kilometer, $\mathrm{v}_{k}$ is a spatially unstructured random effect that is assumed to be normally distributed with mean zero and variance $\sigma_{v}{ }_{v}^{2}$ and $\mathrm{u}_{k}$ is spatially structured random effect that was modeled using a conditional autoregressive (CAR) prior $\mathrm{u}_{k} \sim \mathrm{N}\left(\overline{\mathrm{u}}_{\sim k}, \sigma_{u}{ }^{2} / \mathrm{n}_{k}\right)$, where $-\mathrm{k}$ denotes the neighbors of the kth district based on a simple adjacency matrix and $\mathrm{n}_{k}$ is the corresponding number of neighbors.

SUPPLEMENTARY TABLE S1. Spearman correlation between pneumonia and socioecological covariates in Children Under Five-Years Old — Bangladesh, 2012-2016.

\begin{tabular}{|c|c|c|c|c|c|c|c|c|c|}
\hline & Variables & 1 & 2 & 3 & 4 & 5 & 6 & 7 & 8 \\
\hline 1 & Pneumonia & - & & & & & & & \\
\hline 2 & Temperature & 0.094 & & & & & & & \\
\hline 3 & Temperature variation & 0.161 & 0.235 & & & & & & \\
\hline 4 & Rainfall & -0.019 & $0.268^{*}$ & -0.146 & & & & & \\
\hline 5 & Education & 0.008 & -0.011 & -0.129 & 0.068 & & & & \\
\hline 6 & Internet use & 0.126 & 0.063 & 0.223 & 0.017 & -0.066 & & & \\
\hline 7 & Population density & -0.148 & $-0.25^{\star}$ & $-0.276^{*}$ & 0.028 & 0.075 & 0.162 & & \\
\hline 8 & Poverty & 0.069 & -0.14 & 0.163 & -0.077 & 0.095 & -0.209 & $-0.336^{*}$ & - \\
\hline
\end{tabular}

Note: - represnt its pneumonia itself, there will be no number.

${ }^{*} P<0.05$.

SUPPLEMENTARY TABLE S2. Descriptive statistics of childhood pneumonia and different socioecological factors Bangladesh, 2012-2016.

\begin{tabular}{ccc}
\hline Variables & Mean \pm SD & Range \\
\hline Pneumonia & $747.82 \pm 245.32$ & $355.53-1612.10$ \\
Temperature $\left({ }^{\circ} \mathrm{C}\right)$ & $30.97 \pm 0.48$ & $29.50-31.98$ \\
Temperature variation $\left({ }^{\circ} \mathrm{C}\right)$ & $3.63 \pm 0.55$ & $2.00-4.99$ \\
Rain $(\mathrm{mm})$ & $164.54 \pm 101.37$ & $3.13-386.96$ \\
Education $(\%)$ & $54.66 \pm 7.75$ & $37.50-73.70$ \\
Internet use $(\%)$ & $0.62 \pm 0.76$ & $0.14-6.03$ \\
Poverty incidence $(\%)$ & $27.45 \pm 15.31$ & $2.60-70.80$ \\
Under five years population density (per square $\mathrm{km})$ & $117.72 \pm 87.05$ & $10.81-656.54$ \\
\hline
\end{tabular}


SUPPLEMENTARY TABLE S3. Model comparison for relative risk of monthly childhood pneumonia, underlying socioecological factors, and different random effects - Bangladesh, 2012-2016.

\begin{tabular}{cccc}
\hline Model & Random effect & Deviance Information Criterion (DIC) & Effective number of parameters (pD) \\
\hline Model I & No & 19954.20 & 30.146 \\
Model II & No & 17382.41 & 5.001 \\
Model III & No & 13773.00 & 11.134 \\
Model IV & $u$ & 665.98 & 63.940 \\
Model V & $v$ & 665.67 & 63.812 \\
Model VI & $u$ and $v$ & 665.47 & 63.719 \\
\hline
\end{tabular}

SUPPLEMENTARY TABLE S4. List of high-risk districts of Bangladesh for childhood pneumonia from 2012 to 2016.

\begin{tabular}{ccc}
\hline Name of the district & Relative Risk (95\% Credible interval) & Location \\
\hline Rangamati & $5.97(5.63-6.31)$ & South-eastern \\
Pirojpur & $4.71(4.48-4.93)$ & South-western \\
Jhalkathi & $4.38(4.09-4.66)$ & South-western \\
Jaipurhut & $3.95(3.70-4.19)$ & Sorth-eastern \\
Bandarbon & $3.77(3.48-4.07)$ & South-eastern \\
Meherpur & $3.50(3.23-3.78)$ & Central \\
Rajbari & $3.31(3.11-3.50)$ & South-eastern \\
Khagrachari & $3.25(3.02-3.49)$ & Northern \\
Panchagarh & $2.96(2.78-3.14)$ & Sern \\
\hline
\end{tabular}

